Review Article

\title{
Inflammatory Cytokine Signaling during Development of Pancreatic and Prostate Cancers
}

\author{
Geou-Yarh Liou \\ Center for Cancer Research \& Therapeutic Development and Department of Biological Sciences, Clark Atlanta University, \\ 223 James P. Brawley Drive SW, Atlanta, GA 30314, USA \\ Correspondence should be addressed to Geou-Yarh Liou; gliou@cau.edu
}

Received 28 July 2017; Revised 31 October 2017; Accepted 8 November 2017; Published 12 December 2017

Academic Editor: Shi Jin

Copyright (c) 2017 Geou-Yarh Liou. This is an open access article distributed under the Creative Commons Attribution License, which permits unrestricted use, distribution, and reproduction in any medium, provided the original work is properly cited.

\begin{abstract}
Inflammation is essential for many diseases including cancer. Activation and recruitment of immune cells during inflammation result in a cytokine- and chemokine-enriched cell environment, which affects cancer development. Since each type of cancer has its unique tumor environment, effects of cytokines from different sources such as tumor-infiltrating immune cells, stromal cells, endothelial cells, and cancer cells on cancer development can be quite complex. In this review, how immune cells contribute to tumorigenesis of pancreatic and prostate cancers through their secreted cytokines is discussed. In addition, the cytokine signaling that tumor cells of pancreatic and prostate cancers utilize to benefit their own survival is delineated.
\end{abstract}

\section{Introduction}

Cancers develop because of a dysfunctional immune system of the body, which is unable to detect or eliminate precancerous cells at an early stage. Hijacking the immune system, which eliminates pathogens and unwanted cells such as senescent, damaged, or immature cells under normal physiological conditions, is a very common strategy that cancers utilize for benefitting their long-term growth and survival against locally limited resources, for example, less oxygen in solid tumor tissues. To overcome this shortage of resource, cancer cells express several cytokines, growth factors, and receptors for cytokines and growth factors to become independent of the mitogens that are supplied other than themselves. Another mechanism, which cancer cells utilize, is to recruit immune cells such as macrophages, neutrophils, B cells, and so on, and these tumor-infiltrating immune cells interact with cancer cells, cancer-associated fibroblasts, and themselves. Meanwhile, tumor-associated immune cells secrete certain cytokines, chemokines, and proteases, for example, TGF $\beta$ to dampen T cells, natural killer (NK) cells, and dendritic cells; all of which are engaged in eradicating cancer cells. The indirect effects of immune cells among themselves also create a tumor-favorable outcome. For example, IL- 10 secreted by $\gamma \delta$ Treg cells diminished the cytotoxic activity of $\mathrm{CD} 8^{+} \mathrm{T}$ cells and NK cells, resulting in tumor growth [1]. It is certainly complex that how different types of tumor-infiltrating immune cells affect their biological functions among each other through self-secreted cytokines and chemokines, leading to specific and unique tumor environments in different types of cancer. Moreover, the neighboring cell types around cancer cells also contribute to distinct tumor environments in different types of cancer. For example, breast cancer is neighbored by adipose tissue; pancreatic cancer is accompanied by massive desmoplasia. Given that the same cytokine can result in opposite biological effects in different types of cancer, that is, tumor promoting versus tumor inhibiting due to their unique tumor environment, it is important to comprehensively review cytokine signaling and its functions during cancer development in each type of cancer for shedding light on cancer therapies by altering the tumor environment. In this review, I will mainly focus on cytokine signaling of tumor-facilitated immune cells and of cancer cells that lead to tumor initiation, progression, and metastasis of pancreatic cancer and prostate cancer. 


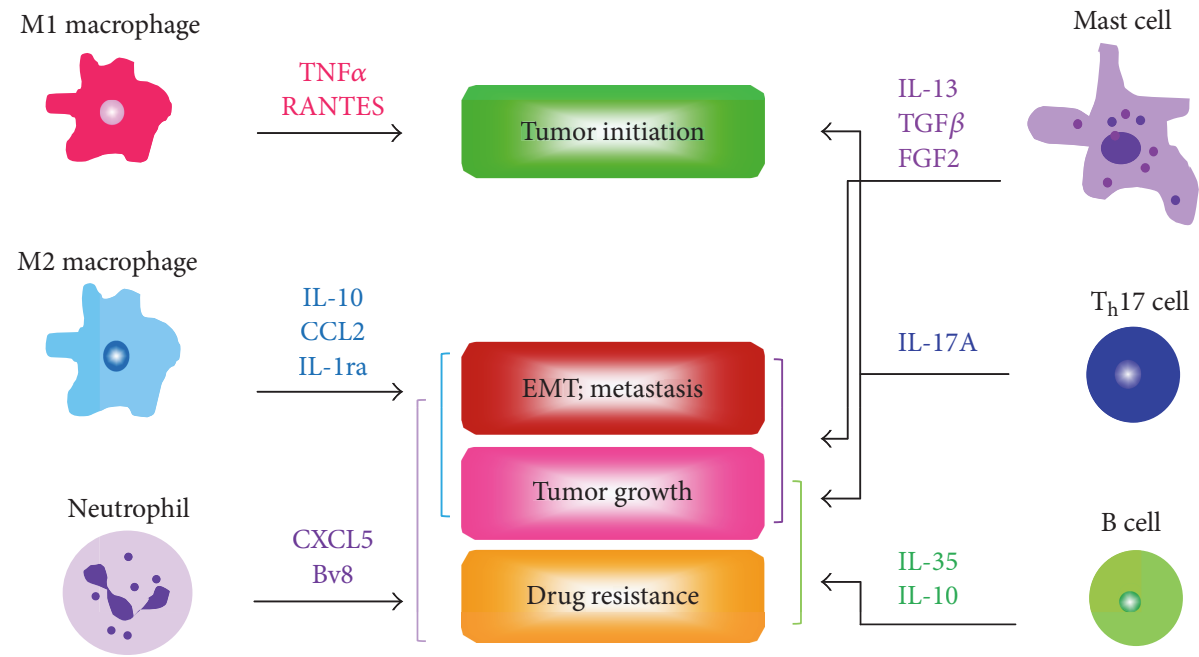

Figure 1: Effects of the cytokines secreted by different types of immune cells on tumor initiation, progression, and dissemination. Cytokines secreted by macrophages, neutrophils, mast cells, $\mathrm{T}_{\mathrm{h}} 17$ cells, and B cells directly lead to initiation and development of pancreatic and prostate cancers. IL: interleukin; RANTES: regulated on activation, normal T cell expressed and secreted; TGF $\beta$ : transforming growth factor $\beta$; FGF2: fibroblast growth factor 2; EMT: epithelial mesenchymal transition.

\section{Pancreatic and Prostate Cancers}

Pancreatic cancer is the most lethal type of cancer with an approximately $7 \% 5$-year survival rate and is projected to become the 2nd leading cause of all cancer-related death by 2030. Pancreatic ductal adenocarcinoma (PDAC) represents over $90 \%$ of pancreatic cancer cases. Oncogenic Kras mutations are present in almost all PDAC patients and are required to initiate and develop PDAC. Another unique feature of PDAC is severe desmoplasia/fibrosis, which occurs at the very early stage of the disease. Pancreatic stellate cells, fibroblasts, and enriched extracellular matrix (ECM) components from desmoplasia orchestrate with other types of cells including immune cells and endothelial cells to promote pancreatic cancer growth and survival.

Prostate cancer is the most common type of cancer in men. In addition, it is also the 2nd leading cause of cancer fatality in men. Unlike PDAC, which is a genetic disorder of oncogenic Kras, key genetic mutations lead to prostate cancer remain unclear. Although several genetically engineered mouse models are generated for studying prostate cancer initiation, development, and dissemination in vivo, none of them fully recapitulates this disease in humans. Most in vivo studies, especially for testing potential therapeutic drugs on prostate cancer, mainly rely on xenograft mouse models using human prostate cancer cell lines or patient tissue samples. It restrains the advancements of how these drugs also affect immune cells and stromal cells, which can contribute to drug efficacy during cancer therapy.

Although pancreatic and prostate cancers are quite different and face different challenges for cancer therapies, the patients of these two cancers share certain features among other cancer patients which include (1) late-onset diseases (in patients' late 60), (2) a gender preference which males rather than females are prone to the diseases, and (3) an ethnic preference which African Americans rather than other racial groups are more susceptible to these cancers. Of note, both cancer types are tightly associated with old age, implicating a pivotal role of aging-related inflammation in these two types of cancer. Herein, an overview of how the cytokine signaling from immune cells and cancer cells affects tumorigenesis of the pancreas and the prostate is provided below.

\section{Cytokine Signaling from Immune Cells for Modulating Tumorigenesis}

The immune system is consisted of many types of immune cells, organs, and antibodies to protect and defend our body. Immune cells can be majorly categorized to phagocytes and lymphocytes which secrete cytokines and chemokines to alter a local cell microenvironment. It has been demonstrated that several cytokines from both phagocytes and lymphocytes potentiate tumor initiation, proliferation, and metastasis of pancreatic and prostate cancers (Figure 1). How these cytokines secreted by different types of immune cells to directly influence cancer cells or indirectly affect nearby immune cells during cancer development are described below in details.

3.1. Cytokines Secreted by Phagocytes and Their Functions. Phagocytes include macrophages, neutrophils, mast cells, and dendritic cells and are capable of ingesting microorganisms, cellular debris, and foreign particles. A large and convincing body of evidence demonstrates the increased infiltrating macrophages and their role in cancer progression and dissemination of PDAC and prostate adenocarcinoma. IL-6 from infiltrating macrophages in the pancreata of PDAC transgenic mice activated transcription factor STAT3 in pancreatic tumor cells, promoting tumor development [2]. Macrophages are categorized to M1 and M2 subtypes, which are also known as classically activated (M1) and alternatively activated (M2) according to their activations (see details in review [3]). M1 macrophages are activated by lipopolysaccharide (LPS), interferon gamma (IFN $\gamma$ ), virus, and so on 
and participate in killing pathogens and clearing up dying cells to protect the host, known as inflammation. During this process, M1 macrophages secrete inflammatory cytokines and generate nitric oxide (NO). However, M2 macrophages stimulated by interleukin-4 (IL-4), IL-13, IL-10, and so on produce extracellular matrix components, angiogenic and chemotactic factors, and IL-10. M2 macrophages are engaged in wound healing, tissue remodeling, allergy, and immunoregulation. Based on the characters of M1 and M2 macrophages described above, several markers such as iNOS, CD38 (for M1 macrophages), Egr2, and CD163 (for M2 macrophages) have been utilized for studying their functions on cancer initiation, development, and dissemination [4-6]. Increased numbers of M2-polarized tumor-associated macrophages (TAM) were reported to correlate with a poor prognosis of PDAC [7, 8]. Of note, M1 proinflammatory macrophages, which generally are thought to be antitumor, have been demonstrated to initiate PDAC development [9-11]. TNF $\alpha$ and RANTES secreted by M1 macrophages upregulate expressions and activities of matrix metalloproteinase 9 (MMP9) of pancreatic acini, resulting in transdifferentiating these cells to a progenitor duct-like phenotype, which later can further become PDAC cells. This piece of evidence also provides a plausible and common scenery of how inflammation-related conditions such as pancreatitis, obesity, diabetes, aging, and so on increase the risk of getting PDAC.

In a pdx ${ }^{\text {cre }}: \mathrm{Kras}^{\mathrm{G} 12 \mathrm{D}}: \mathrm{Trp} 53^{\mathrm{R} 172 \mathrm{H}}$ (KPC) transgenic mouse model that recapitulates human metastatic PDAC, depletion of macrophages by pharmacological compound clodronate liposomes at an early stage of PDAC reduced metastatic cancer cells in the liver and lungs [12]. M2polarized macrophages secreted high levels of CCL2 and IL-1ra around preinvasive pancreatic intraepithelial neoplasia (PanIN) lesions in vivo, resulting in elevated proliferation of PanIN cells through enhanced ERK signaling [13]. In addition, prevention of macrophage polarization to a M2 subtype by an IL-13 neutralizing antibody diminished fibrosis, which is associated with pancreatic tumor proliferation and progression. In a coculture system, in addition to elevated cancer cell proliferation and migration, IL-4-polarized M2 macrophages also induced epithelialmesenchymal transition (EMT) in human pancreatic cancer cell lines [14]. Furthermore, depletion of toll-like receptor 4 (TLR4) or neutralization of IL-10 in M2 macrophages blocked the EMT ability of cancer cells. Very recently, it has been demonstrated that inflammasome component protein NLRP3 expressed by M2 macrophages $\left(\mathrm{CD}^{206}{ }^{+} \mathrm{MHCII}^{-}\right)$is essential for their proliferation in PDAC [15]. In addition, these NLRP $3^{+}$macrophages also resulted in increased populations of $\mathrm{T}_{\mathrm{h}} 17$ and Treg cells and an inactivation of cytotoxic $\mathrm{CD} 8^{+} \mathrm{T}$ cells. Interference with inflammasome by targeting its component proteins through pharmacological compounds or NLRP3 knockout reduced PDAC formation in vivo.

Accumulating evidence indicated an increased recruitment of M2 immunosuppressive macrophages in prostate cancer, associating with cancer growth, metastasis, and drug resistance, thus leading to worse outcomes for prostate cancer patients [16-20]. However, to date, research studies in prostate cancer field majorly are focused on how prostate cancer affects macrophage polarization and infiltration due to several discrepant clinical data of inflammation/ macrophages in prostate cancer, which may be due to varied markers of different immune cell types and quality of specimens.

Several lines of evidence have demonstrated involvement of neutrophils in cancer initiation, progression, and dissemination. These processes are mediated by neutrophil-derived products including cytokine, chemokines, proteases, reactive oxygen species (ROS), and so on (see detailed reviews in $[21,22])$. However, the detailed mechanisms of how these tumor-associated neutrophils (TAN) promote cancer development and metastasis, especially in varied tumor environments of different types of cancer, remain unclear. A neutrophil-expressed glycoprotein lipocalin 2, known for combatting bacterial infection, was present in preinvasive PanIN lesions and PDAC and was suggested as a marker for early diagnosis of PDAC [23]. In addition, the ratio of neutrophil to lymphocyte found in the peripheral blood was correlated to the overall survival of PDAC patients $[24,25]$. TAN secretes several cytokines and chemokines such as CXCL5, TGF $\beta$, and TNF during tumor development and metastasis. In transgenic KPC mice of PDAC, high levels of CXCL5 in the tumor stroma were detected [26]. Moreover, knockout of CXCL5 receptor CXCR2 in PDAC cells dramatically blocked liver metastases. In a xenograft mouse model of human pancreatic cancer AsPC-1 cells, administering an inhibitor of chemokine Bv8, which is secreted by TAN, reduced tumor size and enhanced gemcitabine-induced cytotoxicity of cancer cells [27].

Mast cells participate in many physiological processes such as immune tolerance, angiogenesis, wound healing, and defense against pathogens. Dysregulation of mast cell activation associates with many disorders including cancer. Increased infiltration of mast cells was reported in PDAC patient samples and correlates with a high grade of tumor as well as a poor prognosis [28, 29]. Implantation of murine cancer Pan02 cells into the pancreas tissues of mast celldeficient mice failed to develop PADC in vivo [30]. It has been shown that neutralization of IL-13 secreted by mast cells hindered proliferation of human pancreatic stellate cells (PSC) [31]. Interestingly, blockade of IL-13 also decreased TGF $\beta$ expression and activation of Smad2 in PSC, suggesting that TGF $\beta$ signaling as downstream of IL-13 promotes PSC proliferation.

Similar to that in PDAC patients, more mast cells were present in prostate adenocarcinoma tissues and further increased their recruitment to castrate-resistant prostate tumors [32]. Less mast cells present within prostate tumors may indicate a high recurrence rate of the disease [33]. In an orthotopic rat model of prostate cancer, coinjection of mast cells with cancer AT-1 cells increased tumor growth [32]. Moreover, increased mRNA levels and protein expressions of fibroblast growth factor 2 (FGF2) were detected in mast cell-infiltrating prostate tumors. In a xenograft mouse model, coimplantation of human prostate cancer cells with mast cells enhanced tumor metastasis to the diaphragm 
[34]. In addition, cultured mast cells downregulated mRNA levels of androgen receptor of human prostate cancer cell lines, leading to increased invasiveness and migration ability of cancer cells.

3.2. Cytokines Secreted by Lymphocytes and Their Functions. Lymphocytes include T cells, B cells, and natural killer (NK) cells. Dysfunctional T cells generated through different molecular and cellular mechanisms such as $\mathrm{T}$ cell anergy, exhaustion, and senescence allow tumor growth by escaping immune surveillance. In addition, presence of regulatory $\mathrm{T}$ cells (Treg), which suppress self-activation of $\mathrm{T}$ cells, also contributes to immunosuppressive tumor microenvironments, blocking antitumor immune responses, and subsequent cancer progression. In tumor tissue samples of PDAC patients, more $\mathrm{CD} 4^{+} \mathrm{CD} 25^{+} \mathrm{Foxp} 3^{+}$Treg cells were detected and linked to a poor prognosis $[35,36]$. This increase in Treg cells also correlates with less cytotoxic $\mathrm{CD}^{+} \mathrm{T}$ cells and $\mathrm{CD}^{+}{ }^{+} \mathrm{T}$ helper cells in PDAC and precancerous PanIN lesions. Reprogramming of Treg cells by a CD25 neutralizing antibody daclizumab results in an increase in $\mathrm{CD} 56^{\text {bright }}$ natural killer cells and functional $\mathrm{T}$ cells such as cytotoxic $\mathrm{CD} 8^{+} \mathrm{T}$ cells and $\mathrm{CD} 4^{+} \mathrm{T}$ helper cells in a small clinical trial of metastatic breast cancer patients who receive a cancer vaccine [37]. Results from a heterotopic mouse model of PDAC using nonmetastatic murine Pan02 cancer cells and an orthotopic xenograft mouse model using metastatic human Panc-1 cancer cells revealed that pancreatic cancer expresses CCL5 to recruit CD $4^{+}$Foxp $3^{+}$Treg cells, which have high levels of CCR5 $[38,39]$. Interruption of this interaction between CCL5 and CCR5 by knocking down CCL5 in cancer cells that were used for cancer cell transplantation in mice impedes cancer growth. This suppression effect can also be achieved by administering a CCR5 inhibitor TAK-779- or a CCL5-neutralizing antibody in mice. IL- 10 secreted by $\mathrm{CD} 4^{+}$Foxp $3^{+}$Treg cells renders $\mathrm{T}$ cell anergy (unresponsive to IL-10 restimulations) (see review in $[40,41])$. IL-10 from Treg can also prevent T cell expansion by directly suppressing IL-2 production in T cells. In addition to induction of IL-10 expression in Foxp $3^{+}$Treg cells, TGF $\beta$ from $\mathrm{CD}^{+}{ }^{+} \mathrm{Foxp}^{+}{ }^{+}$Treg cells and pancreatic cancer also stimulates transcription factor Foxp3 expression in Foxp $3^{-}$ naïve $\mathrm{T}$ cells, leading to differentiation of Foxp $3^{+}$Treg cells $[42,43]$. Whether targeting Treg cells, for example, through CD25 blockade or Treg-secreted cytokines as mentioned previously is sufficient to overcome immune resistance of pancreatic cancer in vivo remains to be evaluated. In prostate cancer, a high density of Treg cells was detected in cancer tissues and associated with an advanced tumor stage as well as a low survival rate $[44,45]$. In prostate cancer with bone metastasis, Treg cells expressing high levels of CXCR4 were recruited to bone marrow through an interaction with CXCL12, which is enriched in the bone marrow [46]. Meanwhile, the Treg cells in the bone marrow upregulated RANKL expression to increase their proliferation, which is mediated by RNAK ${ }^{+}$dendritic cells.

$\mathrm{T}$ helper $17\left(\mathrm{~T}_{\mathrm{h}} 17\right)$ cells are $\mathrm{T}$ helper cells producing IL-17 and involved in autoimmune and inflammatory disorders. In response to different stimuli including IL-6,
$\operatorname{TGF} \beta$, CCL20, IL-23, IL-1 $\beta$, and so on, especially under cancer microenvironments, Th17 cells can mediate tumor regression or tumor promotion [47-49]. In a PDAC heterograft mouse model, increased infiltration of Th17 cells was observed in IL-6-expressed tumors that cause high mortality in mice [50]. In a transgenic mouse model of PDAC, oncogenic Kras ${ }^{\mathrm{G} 12 \mathrm{D}}$ induced IL17A-expressed $\mathrm{T}_{\mathrm{h}} 17$ cells, leading to tumor initiation and progression of precancerous PanIN lesions [48]. This initiation and promotion effects by $T_{h} 17$ cells are mediated through their interaction with tumorinitiating cells and PanIN cells, both expressing IL-17 receptor A (IL-17RA). In addition, manipulation of IL-17A by overexpression of IL-17A in the pancreas or knockout of IL-17A in hematopoietic cells interfered initiation and development of pancreatic tumor in vivo. In a xenograft mouse model of human pancreatic cancer CFPAC-1 cells, depletion of IL-17RB impedes cancer growth and metastasis to the liver and lungs [51]. The effect of IL-17RB on PDAC proliferation and dissemination was mediated by upregulation of CCL20, CXCL1, and IL-8 cytokines through ERK signaling in PDAC cancer cells. In prostate cancer, more $\mathrm{T}_{\mathrm{h}} 17$ cells and higher levels of its secreted cytokine IL-17A are linked to a worse outcome of patients [52, 53]. Knockout of IL-17RC, a receptor for IL-17A, decreased invasive prostate tumor growth of $\mathrm{PTEN}^{-1-}$ transgenic mice through downregulation of MMP7 and increased expressions of MMP inhibitors TIMP1, 2, and 4 [54]. Interference with $\mathrm{T}_{\mathrm{h}} 17$ cells by administration of SR1001, a small molecule inhibitor targeting $\mathrm{T}_{\mathrm{h}} 17$ transcription factors $\mathrm{ROR} \gamma \mathrm{t}$ and $\operatorname{ROR} \alpha$, or with $\mathrm{T}_{\mathrm{h}} 17$ cell-secreted IL17 by an IL-17neutralizing antibody, results in reduced cancer proliferation, angiogenesis, and inflammation of $\mathrm{PTEN}^{-/-}$mouse prostate tissues [55].

Microarray study using human samples of PDAC demonstrated increased mRNA expressions of CD20, a marker for B cells [56], suggesting an association of B cells with PDAC development. Infiltration of protumorigenic $B$ cells is associated with hypoxia and fibroblast-enriched stroma [57, 58]. Depletion of B cells, such as use of a CD20 neutralizing antibody or B cell-deficient mice, significantly delays the growth of PDAC and its precursor pancreatic intraepithelial neoplasia (PanIN) in transgenic and orthotopic mouse models [56-58]. Furthermore, reconstitution of functional B cells obtained from wildtype mice through adoptive transfer method rescued pancreatic tumor growth. Among these protumorigenic B cells, which are $\mathrm{CD} 1 \mathrm{~d}^{\mathrm{hi}} \mathrm{CD}^{+}$, increased levels of IL-35 were observed and speculated to be responsible for pancreatic tumor growth [58].

Although an increased density of B cell within prostate tumors has been reported in prostate cancer patient samples $[59,60]$, so far, only a handful publications indicate the function of tumor-infiltrating B cells on tumorigenesis of prostate cancer. In castration-resistant prostate cancer from the xenograft mice, release of IL- $1 \alpha$ from the necrotic primary cancer cells results in CXCL3 secretion, which, in turn, recruits B cells. These tumor-infiltrating B cells produce lymphotoxin to stimulate cancer growth under hormone-independent conditions [61]. In Oxaliplatin-resistant prostate cancers of 


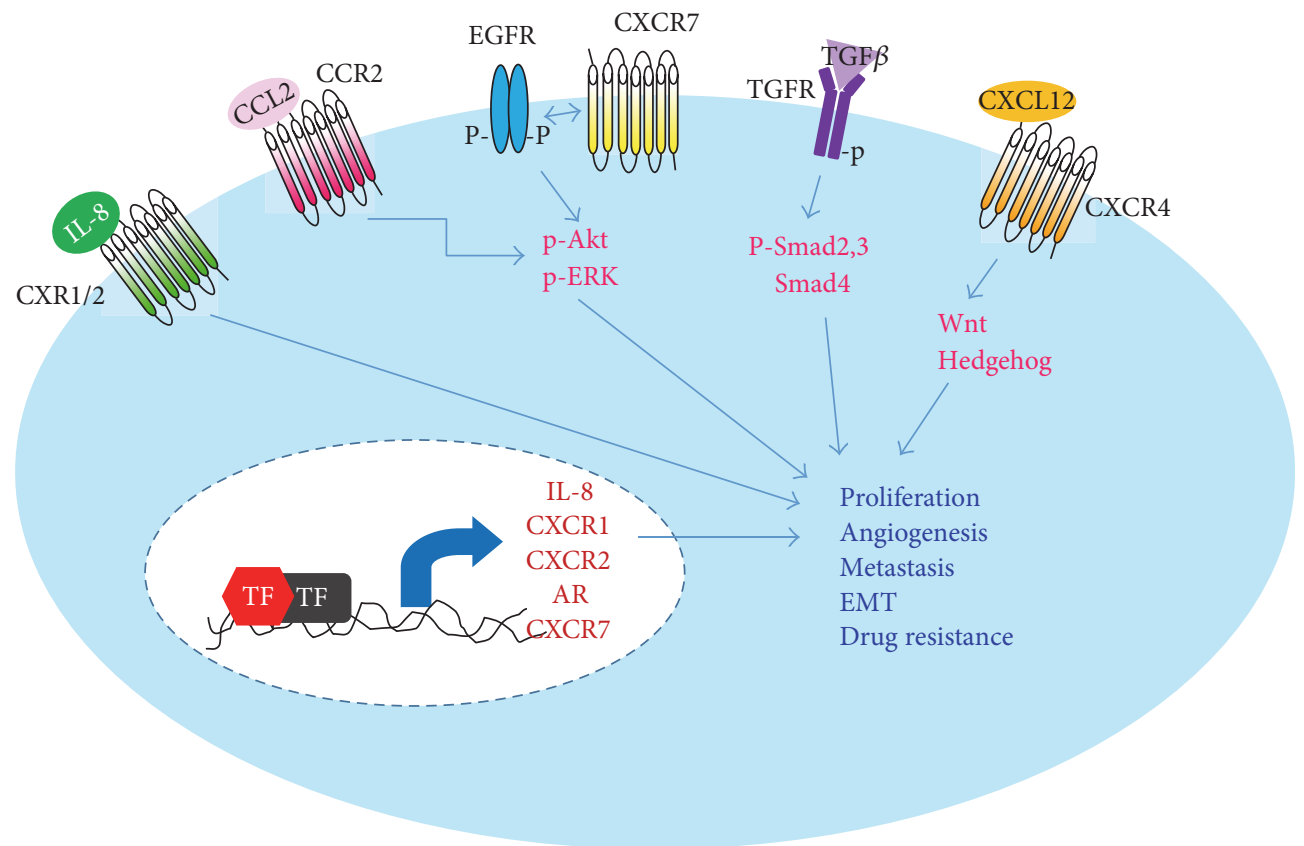

FIGURE 2: Cytokine signaling of cancer cells on modulation of cell proliferation, angiogenesis, metastasis, and drug resistance. Cytokine signaling pathways utilized by pancreatic and prostatic tumor cells through an autocrine-signaling mechanism to elevate their own growth, angiogenesis, and drug resistance. In addition, upregulation of these signaling molecules also renders tumor cells a more mesenchymal-like phenotype, which in turn, promotes metastasis. TF: transcription factor; IL-8: interleukin-8; AR: androgen receptor; EMT: epithelial mesenchymal transition; p: phosphorylated.

TRAMP transgenic mice, increased numbers of B lymphocytes were detected [59]. These infiltrating B cells expressed high levels of IL-10, IgA, and programmed death ligand 1 (PD-L1), leading to the development of oxaliplatin drug resistance over time. Moreover, ablation of IL-10, PD-L1, or B cells reinstated prostate cancer sensitivity to Oxaliplatininduced cell death.

\section{Cytokine Signaling from Tumor Cells for Modulating Tumor Growth and Proliferation}

A large and still continuously increasing body of evidence demonstrated that cancer cells upregulate expressions of numerous cytokines to benefit their own survival. This is achieved by modulating surrounding cells to create a tumor-promoting environment. For example, pancreatic tumor cells expressed cytokine IL-13 to repolarize the nearby macrophages to a tumor-promoting/M2 subtype [13]. In addition, cancer cells also express receptors for the upregulated cytokines to persistently support their own growth (known as autocrine-signaling mechanism, see Figure 2) and become more independent on limited exogenous growth factors as well as mitogens. Insights into these cytokine-signaling pathways of cancer cells could shed light on developing a better cancer therapy for PDAC and castrate-resistant prostate adenocarcinoma.

Increased levels of mRNA and protein of IL-8 were detected in human pancreatic cancer cell lines and PDAC patient tissues $([62,63]$; see reviews in [64]). Elevated expression of IL-8 in PDAC tumor under hypoxic conditions associated with cancer metastatic ability in xenograft mice
[65]. In addition, downregulation of IL-8 by expressing a specific antisense oligonucleotide against IL-8 in PDAC cells before implantating in mouse pancreas hindered tumor vascularization, leading to smaller tumors. Upregulation of IL-8 in PDAC is mediated by transcription factors AP-1 and NF- $\kappa$ B; both positively modulate IL- 8 promoter upon oncogenic Kras mutation [66]. Besides, administration of an IL-8-neutralizing antibody, which blocks IL-8 signaling, in xenografted mice resulted in elevated tumor necrosis and decreased angiogenesis without affecting tumor proliferation. In addition to IL-8, its receptors CXCR1 and CXCR2 were also reported in human PDAC Capan-1 cells to regulate cancer cell growth, migration, invasion, and its stem celllike features $[62,63]$. In human androgen-independent prostate adenocarcinoma, stronger expressions of IL-8 were present and correlated with advanced stages of the disease $[67,68]$. Depletion of IL-8 by shRNA technique in cultured prostate cancer cells, which are derived from human castrate-resistant prostate adenocarcinoma, led to reduced cell proliferation and migration and enhanced cytotoxicity in response to chemotherapeutic drugs such as docetaxel [69]. Several signaling pathways involved in potentiating cell proliferation of prostate cancer by IL-8 have been delineated. Increased IL-8 of prostate cancer cells signals through CXCR1 and CXCR2 to induce transcription of androgen receptor (AR) and to activate ERK and Akt, in turn, promoting cancer growth and survival $[68,70]$. In addition, IL-8 of the prostate cancer cells also upregulated EGFRERK signaling through elevating CXCR7 transcripts and protein levels [71]. However, the mitogenic function of CXCR7 in prostate cancer is ligand independent. 
Higher expressions of TGF $\beta$ have been detected in human PDAC tissue samples and associated with a better prognosis of PDAC patients [72-74]. In human pancreatic cancer Panc- 1 cells, results from knockdown of TGF $\beta$ signaling mediators Smad2 and Smad3 demonstrated an opposite function of Smad2 versus Smad3 on TGF $\beta$ induced cell migration [75]. Silencing Smad4 in Panc-1 cells impeded epithelial-to-mesenchymal transition ability of cells (increased E-cadherin; decreased N-cadherin and vimentin), and this alteration of EMT is mediated by loss of nestin, a protein expressed in stem cells during development [76]. In the KPC transgenic mouse model of PDAC, cancer precursors, which are acinar-to-ductal metaplasia (ADM) and PanIN, and PDAC expressed higher levels of activated Smad2 [77]. Moreover, suppression of TGF $\beta$ by a TGF $\beta$-neutralizing antibody in these mice expedited PDAC proliferation and malignant progression and caused death, suggesting an inhibitory effect of TGF $\beta$ during PDAC growth and development. In addition to stromal cells as well as the cells of benign prostatic hyperplasia (BPH) and of prostatic intraepithelial neoplasia (PIN), increased mRNA and protein levels of both TGF $\beta$ and its receptors were detected in cancer cells of human and rat prostate adenocarcinoma tissues $[78,79]$. In an allotransplantation model of rat prostate cancer cells that overexpressed TGF $\beta$, these tumors grew faster and metastasized to the lungs and lymph nodes. Treating these animals with a TGF $\beta$-neutralizing antibody rescued the phenotype [80]. Moreover, when the TGF $\beta$ expressed cancer cells were cultured in vitro in $2 \mathrm{D}$, their proliferation were inhibited as numerous cases reported in cultured human prostate cancer cell lines. This result suggested the importance of cell environment regarding the impact of TGF $\beta$ expressed by cancer cells on their own proliferation and malignancy.

Increased CCL2 expression was present in PDAC tissues and a portion of human PDAC cancer cell lines [13, 81]. Moreover, no CCR2, a receptor for CCL2, was detected in the tested human CCL2-expressed PDAC cell lines. This may be due to a low sensitivity of Northern blotting to detect CCR2 transcripts or cell culture conditions such as 2D culture system versus 3D culture system, hypoxic environment, and so on. Although it is unclear whether tumor cells of PDAC tissues have CCR2, presence of CCR2 was shown in preinvasive PanIN lesions of transgenic KC mice [13]. In addition, in a 3D culture system of murine duct-like cells derived from KC mouse pancreas, exogenous CCL2 treatment promoted cell proliferation through activation of ERK. These results suggested an autocrine mechanism of CCL2 used by pancreatic tumor cells to benefit their growth. Similar to pancreatic cancer, expressions of CCL2 and CCR2 were present in prostate cancer tissues and led to increased cancer proliferation and invasion through Akt signaling $[82,83]$. Interference with this pathway by administration of CCR2 antagonist, CCL2 neutralizing antibody, or PI3 kinase inhibitor or by knockdown of CCL2 in prostate cancer PC3 cells all resulted in decreased tumor formation, smaller tumor size, and less metastases in vivo [82-84].

CXCR4 and its ligand CXCL12 were present in PanIN lesions and PDAC tissues and demonstrated to result in tumor proliferation, cancer progression, angiogenesis, and metastasis [85-87]. CXCR4-CXCL12-caused cell proliferation was mediated via EGFR-Src-PI3 kinase signaling pathway, which subsequently activates ERK [87-89]. For CXCR4-CXCL12-mediated invasion and metastasis of human pancreatic cancer cell lines, activation of Wnt, and Hedgehog signaling pathways were required and led to an EMT phenotype $[90,91]$. Interestingly, inhibition of Wnt, Hedgehog, or CXCL12-CXCR4 signaling by pharmacological compounds resensitized cancer cells to gemcitabine-induced cytotoxicity, suggesting the importance of CXCL12-CXCR4 in PDAC drug resistance [92-94]. Similar to the functions of CXCR4-CXCL12 in PDAC, prostate cancer cells express high levels of CXCR4 and CXCL12 and utilize the same signaling pathways described above to modulate their proliferation, angiogenesis, drug resistance, and metastasis (see review in $[95,96])$.

Cytokine granulocyte-macrophage colony-stimulating factor (GM-CSF) can affect tumor immune response positively or negatively depending on the local tumor microenvironment, which is distinct among cancer types [97]. Accumulating evidence supports a role of GM-CSF in promoting rather than inhibiting the development of pancreatic cancer. Higher expressions of GM-CSF were detected in human and mouse PDAC tissues and have been shown to modulate development of $\mathrm{Gr}-1^{+} \mathrm{CD} 11 \mathrm{~b}^{+}$myeloid cells, which inactivate cytotoxic $\mathrm{CD}^{+} \mathrm{T}$ cells $[98,99]$. In addition, blockade of GM-CSF derived from PDAC by either a GM-CSF-neutralizing antibody or a specific knockdown of GM-CSF in pancreatic cancer cells suppressed tumor growth with less infiltrating $\mathrm{Gr}-1^{+} \mathrm{CD} 11 \mathrm{~b}^{+}$cells in vivo. Interestingly, GM-CSF secreted by cancer-associated mesenchymal stem cells (CA-MSC) was required for cell invasion, metastasis, and transendothelial migration of human pancreatic cancer [100]. In addition, it also modulated EMT and stemness of PDAC. In contrast, despite of unknown mechanism of how GM-CSF inhibits metastatic prostate cancer, GM-CSF has been in the clinical trials for prostate cancer patients since 2001. It has been shown that systemic GMCSF given to the patients in the clinical trials altered immune cell populations with an increase in $\mathrm{CD}^{+} \mathrm{T}$ cells, $\mathrm{CD} 8^{+} \mathrm{T}$ cells, and mature myeloid dendritic cell and a decrease in Treg cells [101]. Interestingly, expressions of receptors for GM-CSF are reported in cultured human prostate carcinoma LNCaP cells [102].

Activation of Fas ligand (FasL) and its receptor (Fas) leads to cell apoptosis. In pancreatic cancer, expressions of FasL and Fas were present in human PDAC tissues and cultured cancer cell lines [103-105]. It has been demonstrated that FasL-expressed PDAC induced apoptosis of the infiltrating lymphoid cells, thus eliminating tumor-killing immune cells [103, 105]. Meanwhile, pancreatic cancer cells were resistant to Fas-induced apoptosis by downregulation of Fas or upregulation of Fas-associated phosphatase 1 (FAP-1), which is mediated by JNK and p38 MAPK [104]. Similar to the functions and expressions of FasL in pancreatic cancer, human prostate cancer cells were also resistant to FasL-induced apoptosis. In addition, soluble FasL (sFasL) was consistently secreted through a cleavage on membrane- 
bound FasL by MMPs in prostate cancer [106]. Tumor exosomes of human prostate carcinoma LNCaP cells expressed FasL and caused cytotoxic $\mathrm{CD}^{+} \mathrm{T}$ cell apoptosis [107]. It has been shown that interaction of FasL with Fas in the intracellular compartment resulted in cancer cell apoptosis of human prostate carcinoma cells that are resistant to anti-Fas antibody CH-11 [108].

\section{Conclusion}

Increased cytokine signaling in both tumor-infiltrating immune cells and cancer cells potentiates tumor growth, metastasis, and drug resistance of pancreatic and prostate cancers. Many cytokines upregulated in cancer cells are mediated through autocrine signaling. Meanwhile, cancer cells also use paracrine-signaling mechanism to recruit immune cells to the tumor site. These infiltrating immune cells then produce more cytokines to directly or indirectly support tumor growth. Certain downstream effectors of cytokines, especially upregulated by cancer cells, modulate stem cell-like properties such as Notch, Wnt, and Hedgehog. Through controlling these pathways, cancer cells are capable of proliferating and surviving even when under harsh conditions, for example, cancer therapy. A comprehensive view of this complex cytokine network among tumor cells, immune cells, and other types of cells including stromal cells, endothelial cells, and so on will provide invaluable information on best strategies to defeat cancer-caused death, for example, use of cytokine inhibitor cocktails in the future.

\section{Conflicts of Interest}

The author declares that there is no conflict of interest regarding the publication of this article.

\section{Acknowledgments}

This work was supported by a grant from the Center for Cancer Research and Therapeutic Development (CCRTD) at Clark Atlanta University funded by NIH G12MD007590 to Geou-Yarh Liou.

\section{References}

[1] N. Seo, Y. Tokura, F. Furukawa, and M. Takigawa, "Downregulation of tumoricidal NK and NK T cell activities by MHC $\mathrm{K}^{\mathrm{b}}$ molecules expressed on Th2-type gammadelta $\mathrm{T}$ and alphabeta $\mathrm{T}$ cells coinfiltrating in early B16 melanoma lesions," Journal of Immunology, vol. 161, no. 8, pp. 41384145, 1998.

[2] M. Lesina, M. U. Kurkowski, K. Ludes et al., "Stat3/Socs3 activation by IL- 6 transsignaling promotes progression of pancreatic intraepithelial neoplasia and development of pancreatic cancer," Cancer Cell, vol. 19, no. 4, pp. 456-469, 2011.

[3] F. O. Martinez and S. Gordon, "The M1 and M2 paradigm of macrophage activation: time for reassessment," F1000Prime Reports, vol. 6, p. 13, 2014.

[4] C. Caux, R. N. Ramos, G. C. Prendergast, N. BendrissVermare, and C. Ménétrier-Caux, "A milestone review on how macrophages affect tumor growth," Cancer Research, vol. 76, no. 22, pp. 6439-6442, 2016.

[5] K. A. Jablonski, S. A. Amici, L. M. Webb et al., "Novel markers to delineate murine M1 and M2 macrophages," PLoS One, vol. 10, no. 12, article e0145342, 2015.

[6] S. R. Nielsen and M. C. Schmid, "Macrophages as key drivers of cancer progression and metastasis," Mediators of Inflammation, vol. 2017, Article ID 9624760, 11 pages, 2017.

[7] Y. C. Hou, Y. J. Chao, H. L. Tung, H. C. Wang, and Y. S. Shan, "Coexpression of CD44-positive/CD133-positive cancer stem cells and CD204-positive tumor-associated macrophages is a predictor of survival in pancreatic ductal adenocarcinoma," Cancer, vol. 120, no. 17, pp. 27662777, 2014.

[8] H. Kurahara, H. Shinchi, Y. Mataki et al., "Significance of M2-polarized tumor-associated macrophage in pancreatic cancer," The Journal of Surgical Research, vol. 167, no. 2, pp. e211-e219, 2011.

[9] G. Y. Liou, H. Döppler, B. Necela et al., "Mutant KRASinduced expression of ICAM-1 in pancreatic acinar cells causes attraction of macrophages to expedite the formation of precancerous lesions," Cancer Discovery, vol. 5, no. 1, pp. 52-63, 2015.

[10] G. Y. Liou, H. Döppler, B. Necela et al., "Macrophagesecreted cytokines drive pancreatic acinar-to-ductal metaplasia through NF-kappaB and MMPs," The Journal of Cell Biology, vol. 202, no. 3, pp. 563-577, 2013.

[11] G. Y. Liou and P. Storz, "Inflammatory macrophages in pancreatic acinar cell metaplasia and initiation of pancreatic cancer," Oncoscience, vol. 2, no. 3, pp. 247-251, 2015.

[12] H. Griesmann, C. Drexel, N. Milosevic et al., "Pharmacological macrophage inhibition decreases metastasis formation in a genetic model of pancreatic cancer," Gut, vol. 66, no. 7, pp. 1278-1285, 2017.

[13] G. Y. Liou, L. Bastea, A. Fleming et al., "The presence of interleukin-13 at pancreatic ADM/PanIN lesions alters macrophage populations and mediates pancreatic tumorigenesis," Cell Reports, vol. 19, no. 7, pp. 1322-1333, 2017.

[14] C. Y. Liu, J. Y. Xu, X. Y. Shi et al., "M2-polarized tumorassociated macrophages promoted epithelial-mesenchymal transition in pancreatic cancer cells, partially through TLR4/IL-10 signaling pathway," Laboratory Investigation, vol. 93, no. 7, pp. 844-854, 2013.

[15] D. Daley, V. R. Mani, N. Mohan et al., "NLRP3 signaling drives macrophage-induced adaptive immune suppression in pancreatic carcinoma," The Journal of Experimental Medicine, vol. 214, no. 6, pp. 1711-1724, 2017.

[16] S. J. Ellem, H. Wang, M. Poutanen, and G. P. Risbridger, "Increased endogenous estrogen synthesis leads to the sequential induction of prostatic inflammation (prostatitis) and prostatic pre-malignancy," The American Journal of Pathology, vol. 175, no. 3, pp. 1187-1199, 2009.

[17] T. Fujii, K. Shimada, O. Asai et al., "Immunohistochemical analysis of inflammatory cells in benign and precancerous lesions and carcinoma of the prostate," Pathobiology, vol. 80, no. 3, pp. 119-126, 2013.

[18] K. Gollapudi, C. Galet, T. Grogan et al., "Association between tumor-associated macrophage infiltration, high grade prostate cancer, and biochemical recurrence after radical prostatectomy," American Journal of Cancer Research, vol. 3, no. 5, pp. 523-529, 2013. 
[19] J. Escamilla, S. Schokrpur, C. Liu et al., "CSF1 receptor targeting in prostate cancer reverses macrophage-mediated resistance to androgen blockade therapy," Cancer Research, vol. 75, no. 6, pp. 950-962, 2015.

[20] M. Lanciotti, L. Masieri, M. R. Raspollini et al., "The role of M1 and M2 macrophages in prostate cancer in relation to extracapsular tumor extension and biochemical recurrence after radical prostatectomy," BioMed Research International, vol. 2014, Article ID 486798, 6 pages, 2014.

[21] S. B. Coffelt, M. D. Wellenstein, and K. E. de Visser, "Neutrophils in cancer: neutral no more," Nature Reviews Cancer, vol. 16, no. 7, pp. 431-446, 2016.

[22] A. D. Gregory and A. M. Houghton, "Tumor-associated neutrophils: new targets for cancer therapy," Cancer Research, vol. 71, no. 7, pp. 2411-2416, 2011.

[23] N. Moniaux, S. Chakraborty, M. Yalniz et al., "Early diagnosis of pancreatic cancer: neutrophil gelatinase-associated lipocalin as a marker of pancreatic intraepithelial neoplasia," British Journal of Cancer, vol. 98, no. 9, pp. 1540-1547, 2008.

[24] I. Bhatti, O. Peacock, G. Lloyd, M. Larvin, and R. I. Hall, "Preoperative hematologic markers as independent predictors of prognosis in resected pancreatic ductal adenocarcinoma: neutrophil-lymphocyte versus platelet-lymphocyte ratio," American Journal of Surgery, vol. 200, no. 2, pp. 197203, 2010.

[25] X. An, P. R. Ding, Y. H. Li et al., "Elevated neutrophil to lymphocyte ratio predicts survival in advanced pancreatic cancer," Biomarkers, vol. 15, no. 6, pp. 516-522, 2010.

[26] C. W. Steele, S. A. Karim, J. D. G. Leach et al., "CXCR2 inhibition profoundly suppresses metastases and augments immunotherapy in pancreatic ductal adenocarcinoma," Cancer Cell, vol. 29, no. 6, pp. 832-845, 2016.

[27] V. F. Curtis, H. Wang, P. Yang et al., "A PK2/Bv8/ PROK2 antagonist suppresses tumorigenic processes by inhibiting angiogenesis in glioma and blocking myeloid cell infiltration in pancreatic cancer," PLoS One, vol. 8, no. 1, article e54916, 2013.

[28] M. Ammendola, R. Sacco, G. Sammarco et al., "Mast cells density positive to tryptase correlates with angiogenesis in pancreatic ductal adenocarcinoma patients having undergone surgery," Gastroenterology Research and Practice, vol. 2014, Article ID 951957, 7 pages, 2014.

[29] M. J. Strouch, E. C. Cheon, M. R. Salabat et al., "Crosstalk between mast cells and pancreatic cancer cells contributes to pancreatic tumor progression," Clinical Cancer Research, vol. 16, no. 8, pp. 2257-2265, 2010.

[30] D. Z. Chang, Y. Ma, B. Ji et al., "Mast cells in tumor microenvironment promotes the in vivo growth of pancreatic ductal adenocarcinoma," Clinical Cancer Research, vol. 17, no. 22, pp. 7015-7023, 2011.

[31] Y. Ma, R. F. Hwang, C. D. Logsdon, and S. E. Ullrich, "Dynamic mast cell-stromal cell interactions promote growth of pancreatic cancer," Cancer Research, vol. 73, no. 13, pp. 3927-3937, 2013.

[32] A. Johansson, S. Rudolfsson, P. Hammarsten et al., "Mast cells are novel independent prognostic markers in prostate cancer and represent a target for therapy," The American Journal of Pathology, vol. 177, no. 2, pp. 1031-1041, 2010.

[33] H. A. Hempel, N. S. Cuka, I. Kulac et al., "Low intratumoral mast cells are associated with a higher risk of prostate cancer recurrence," Prostate, vol. 77, no. 4, pp. 412-424, 2017.
[34] L. Li, Q. Dang, H. Xie et al., "Infiltrating mast cells enhance prostate cancer invasion via altering LncRNA-HOTAIR/ PRC2-androgen receptor (AR)-MMP9 signals and increased stem/progenitor cell population," Oncotarget, vol. 6, no. 16, pp. 14179-14190, 2015.

[35] N. Hiraoka, K. Onozato, T. Kosuge, and S. Hirohashi, "Prevalence of $\mathrm{FOXP}^{+}$regulatory $\mathrm{T}$ cells increases during the progression of pancreatic ductal adenocarcinoma and its premalignant lesions," Clinical Cancer Research, vol. 12, no. 18, pp. 5423-5434, 2006.

[36] Y. Tang, X. Xu, S. Guo et al., "An increased abundance of tumor-infiltrating regulatory $\mathrm{T}$ cells is correlated with the progression and prognosis of pancreatic ductal adenocarcinoma," PLoS One, vol. 9, no. 3, article e91551, 2014.

[37] A. J. Rech, R. Mick, S. Martin et al., "CD25 blockade depletes and selectively reprograms regulatory $\mathrm{T}$ cells in concert with immunotherapy in cancer patients," Science Translational Medicine, vol. 4, no. 134, article 134ra62, 2012.

[38] M. C. Tan, P. S. Goedegebuure, B. A. Belt et al., "Disruption of CCR5-dependent homing of regulatory $\mathrm{T}$ cells inhibits tumor growth in a murine model of pancreatic cancer," Journal of Immunology, vol. 182, no. 3, pp. 1746-1755, 2009.

[39] X. Wang, M. Lang, T. Zhao et al., "Cancer-FOXP3 directly activated CCL5 to recruit FOXP $3^{+}$Treg cells in pancreatic ductal adenocarcinoma," Oncogene, vol. 36, no. 21, pp. 3048-3058, 2017.

[40] D. K. Sojka, Y. H. Huang, and D. J. Fowell, "Mechanisms of regulatory $\mathrm{T}$-cell suppression - a diverse arsenal for a moving target," Immunology, vol. 124, no. 1, pp. 13-22, 2008.

[41] M. Saraiva and A. O'Garra, "The regulation of IL-10 production by immune cells," Nature Reviews. Immunology, vol. 10, no. 3, pp. 170-181, 2010.

[42] C. L. Maynard, L. E. Harrington, K. M. Janowski et al., "Regulatory T cells expressing interleukin 10 develop from Foxp $3^{+}$and Foxp $3^{-}$precursor cells in the absence of interleukin 10," Nature Immunology, vol. 8, no. 9, pp. 931-941, 2007.

[43] T. A. Moo-Young, J. W. Larson, B. A. Belt et al., "Tumorderived TGF-beta mediates conversion of $\mathrm{CD} 4^{+} \mathrm{Foxp}^{+}$ regulatory $\mathrm{T}$ cells in a murine model of pancreas cancer," Journal of Immunotherapy, vol. 32, no. 1, pp. 12-21, 2009.

[44] A. Flammiger, L. Weisbach, H. Huland et al., "High tissue density of FOXP3+ T cells is associated with clinical outcome in prostate cancer," European Journal of Cancer, vol. 49, no. 6, pp. 1273-1279, 2013.

[45] A. M. Miller, K. Lundberg, V. Ozenci et al., "CD $4{ }^{+} C D 25^{\text {high }} \mathrm{T}$ cells are enriched in the tumor and peripheral blood of prostate cancer patients," Journal of Immunology, vol. 177, no. 10, pp. 7398-7405, 2006.

[46] E. Zhao, L. Wang, J. Dai et al., "Regulatory T cells in the bone marrow microenvironment in patients with prostate cancer," Oncoimmunology, vol. 1, no. 2, pp. 152-161, 2012.

[47] N. Martin-Orozco, Y. Li, Y. Wang et al., "Melanoma cells express ICOS ligand to promote the activation and expansion of T-regulatory cells," Cancer Research, vol. 70, no. 23, pp. 9581-9590, 2010.

[48] F. McAllister, J. M. Bailey, J. Alsina et al., "Oncogenic Kras activates a hematopoietic-to-epithelial IL-17 signaling axis in preinvasive pancreatic neoplasia," Cancer Cell, vol. 25, no. 5, pp. 621-637, 2014.

[49] S. Wu, K. J. Rhee, E. Albesiano et al., "A human colonic commensal promotes colon tumorigenesis via activation of 
T helper type $17 \mathrm{~T}$ cell responses," Nature Medicine, vol. 15, no. 9, pp. 1016-1022, 2009.

[50] J. L. Gnerlich, J. B. Mitchem, J. S. Weir et al., "Induction of Th17 cells in the tumor microenvironment improves survival in a murine model of pancreatic cancer," Journal of Immunology, vol. 185, no. 7, pp. 4063-4071, 2010.

[51] H. H. Wu, W. W. Hwang-Verslues, W. H. Lee et al., "Targeting IL-17B-IL-17RB signaling with an anti-IL-17RB antibody blocks pancreatic cancer metastasis by silencing multiple chemokines," The Journal of Experimental Medicine, vol. 212, no. 3, pp. 333-349, 2015.

[52] K. S. Sfanos, T. C. Bruno, C. H. Maris et al., "Phenotypic analysis of prostate-infiltrating lymphocytes reveals TH17 and Treg skewing," Clinical Cancer Research, vol. 14, no. 11, pp. 3254-3261, 2008.

[53] G. E. Steiner, M. E. Newman, D. Paikl et al., "Expression and function of pro-inflammatory interleukin IL-17 and IL-17 receptor in normal, benign hyperplastic, and malignant prostate," Prostate, vol. 56, no. 3, pp. 171-182, 2003.

[54] Q. Zhang, S. Liu, D. Ge et al., "Interleukin-17 promotes formation and growth of prostate adenocarcinoma in mouse models," Cancer Research, vol. 72, no. 10, pp. 25892599, 2012.

[55] Q. Zhang, S. Liu, D. Ge et al., “Targeting Th17-IL-17 pathway in prevention of micro-invasive prostate cancer in a mouse model," Prostate, vol. 77, no. 8, pp. 888-899, 2017.

[56] A. J. Gunderson, M. M. Kaneda, T. Tsujikawa et al., "Bruton tyrosine kinase-dependent immune cell cross-talk drives pancreas cancer," Cancer Discovery, vol. 6, no. 3, pp. 270285, 2016.

[57] K. E. Lee, M. Spata, L. J. Bayne et al., "Hif1a deletion reveals pro-neoplastic function of B cells in pancreatic neoplasia," Cancer Discovery, vol. 6, no. 3, pp. 256-269, 2016.

[58] Y. Pylayeva-Gupta, S. Das, J. S. Handler et al., "IL35producing $\mathrm{B}$ cells promote the development of pancreatic neoplasia," Cancer Discovery, vol. 6, no. 3, pp. 247-255, 2016.

[59] S. Shalapour, J. Font-Burgada, G. di Caro et al., "Immunosuppressive plasma cells impede T-cell-dependent immunogenic chemotherapy," Nature, vol. 521, no. 7550, pp. 94-98, 2015.

[60] J. R. Woo, M. A. Liss, M. T. Muldong et al., "Tumor infiltrating B-cells are increased in prostate cancer tissue," Journal of Translational Medicine, vol. 12, no. 1, p. 30, 2014.

[61] M. Ammirante, J. L. Luo, S. Grivennikov, S. Nedospasov, and M. Karin, "B-cell-derived lymphotoxin promotes castrationresistant prostate cancer," Nature, vol. 464, no. 7286, pp. 302-305, 2010.

[62] L. Chen, J. Fan, H. Chen et al., "The IL-8/CXCR1 axis is associated with cancer stem cell-like properties and correlates with clinical prognosis in human pancreatic cancer cases," Scientific Reports, vol. 4, p. 5911, 2014.

[63] H. Takamori, Z. G. Oades, R. C. Hoch, M. Burger, and I. U. Schraufstatter, "Autocrine growth effect of IL-8 and GROalpha on a human pancreatic cancer cell line, Capan1," Pancreas, vol. 21, no. 1, pp. 52-56, 2000.

[64] Y. Y. Yako, D. Kruger, M. Smith, and M. Brand, "Cytokines as biomarkers of pancreatic ductal adenocarcinoma: a systematic review," PLoS One, vol. 11, no. 5, article e0154016, 2016.

[65] Q. Shi, J. L. Abbruzzese, S. Huang, I. J. Fidler, Q. Xiong, and K. Xie, "Constitutive and inducible interleukin 8 expression by hypoxia and acidosis renders human pancreatic cancer cells more tumorigenic and metastatic," Clinical Cancer Research, vol. 5, no. 11, pp. 3711-3721, 1999.

[66] A. Sparmann and D. Bar-Sagi, "Ras-induced interleukin-8 expression plays a critical role in tumor growth and angiogenesis," Cancer Cell, vol. 6, no. 5, pp. 447-458, 2004.

[67] S. Araki, Y. Omori, D. Lyn et al., "Interleukin-8 is a molecular determinant of androgen independence and progression in prostate cancer," Cancer Research, vol. 67, no. 14, pp. 68546862, 2007.

[68] C. Murphy, M. McGurk, J. Pettigrew et al., "Nonapical and cytoplasmic expression of interleukin-8, CXCR1, and CXCR2 correlates with cell proliferation and microvessel density in prostate cancer," Clinical Cancer Research, vol. 11, no. 11, pp. 4117-4127, 2005.

[69] R. K. Singh and B. L. Lokeshwar, "Depletion of intrinsic expression of Interleukin-8 in prostate cancer cells causes cell cycle arrest, spontaneous apoptosis and increases the efficacy of chemotherapeutic drugs," Molecular Cancer, vol. 8, no. 1, p. $57,2009$.

[70] A. Seaton, P. Scullin, P. J. Maxwell et al., "Interleukin-8 signaling promotes androgen-independent proliferation of prostate cancer cells via induction of androgen receptor expression and activation," Carcinogenesis, vol. 29, no. 6, pp. 1148-1156, 2008.

[71] R. K. Singh and B. L. Lokeshwar, "The IL-8-regulated chemokine receptor CXCR7 stimulates EGFR signaling to promote prostate cancer growth," Cancer Research, vol. 71, no. 9, pp. 3268-3277, 2011.

[72] G. Bellone, C. Smirne, F. A. Mauri et al., "Cytokine expression profile in human pancreatic carcinoma cells and in surgical specimens: implications for survival," Cancer Immunology, Immunotherapy, vol. 55, no. 6, pp. 684-698, 2006.

[73] K. Hashimoto, Y. Nio, S. Sumi et al., "Correlation between TGF-beta1 and p21 (WAF1/CIP1) expression and prognosis in resectable invasive ductal carcinoma of the pancreas," Pancreas, vol. 22, no. 4, pp. 341-347, 2001.

[74] H. Yue, B. Yang, H. Zhang et al., "Clinical significance of TGF-beta1 and beta-glucuronidase synchronous detection in human pancreatic cancer," Hepatobiliary \& Pancreatic Diseases International, vol. 1, no. 2, pp. 309-311, 2002.

[75] H. Ungefroren, S. Groth, S. Sebens, H. Lehnert, F. Gieseler, and F. Fändrich, "Differential roles of Smad2 and $\operatorname{Smad} 3$ in the regulation of TGF-betal-mediated growth inhibition and cell migration in pancreatic ductal adenocarcinoma cells: control by Rac1," Molecular Cancer, vol. 10, no. 1, p. 67, 2011.

[76] H. T. Su, C. C. Weng, P. J. Hsiao et al., "Stem cell marker nestin is critical for TGF-beta1-mediated tumor progression in pancreatic cancer," Molecular Cancer Research, vol. 11, no. 7, pp. 768-779, 2013.

[77] A. F. Hezel, V. Deshpande, S. M. Zimmerman et al., “TGFbeta and alphavbeta6 integrin act in a common pathway to suppress pancreatic cancer progression," Cancer Research, vol. 72, no. 18, pp. 4840-4845, 2012.

[78] M. R. Cardillo, E. Petrangeli, L. Perracchio, L. Salvatori, L. Ravenna, and F. di Silverio, "Transforming growth factor-beta expression in prostate neoplasia," Analytical and Quantitative Cytology and Histology, vol. 22, no. 1, pp. 110, 2000.

[79] M. S. Steiner, Z. Z. Zhou, D. C. Tonb, and E. R. Barrack, "Expression of transforming growth factor-beta 1 in prostate cancer," Endocrinology, vol. 135, no. 5, pp. 2240-2247, 1994. 
[80] M. S. Steiner and E. R. Barrack, "Transforming growth factorbeta 1 overproduction in prostate cancer: effects on growth in vivo and in vitro," Molecular Endocrinology, vol. 6, no. 1, pp. 15-25, 1992.

[81] P. Monti, B. E. Leone, F. Marchesi et al., "The CC chemokine MCP-1/CCL2 in pancreatic cancer progression: regulation of expression and potential mechanisms of antimalignant activity," Cancer Research, vol. 63, no. 21, pp. 7451-7461, 2003.

[82] Y. Lu, Z. Cai, D. L. Galson et al., "Monocyte chemotactic protein-1 (MCP-1) acts as a paracrine and autocrine factor for prostate cancer growth and invasion," Prostate, vol. 66, no. 12, pp. 1311-1318, 2006.

[83] Y. Lu, Q. Chen, E. Corey et al., "Activation of MCP-1/CCR2 axis promotes prostate cancer growth in bone," Clinical \& Experimental Metastasis, vol. 26, no. 2, pp. 161-169, 2009.

[84] R. D. Loberg, C. Ying, M. Craig et al., “Targeting CCL2 with systemic delivery of neutralizing antibodies induces prostate cancer tumor regression in vivo," Cancer Research, vol. 67, no. 19, pp. 9417-9424, 2007.

[85] T. Koshiba, R. Hosotani, Y. Miyamoto et al., "Expression of stromal cell-derived factor 1 and CXCR4 ligand receptor system in pancreatic cancer: a possible role for tumor progression," Clinical Cancer Research, vol. 6, no. 9, pp. 35303535, 2000.

[86] D. Saur, B. Seidler, G. Schneider et al., "CXCR4 expression increases liver and lung metastasis in a mouse model of pancreatic cancer," Gastroenterology, vol. 129, no. 4, pp. 12371250, 2005.

[87] R. M. Thomas, J. Kim, M. P. Revelo-Penafiel, R. Angel, D. W. Dawson, and A. M. Lowy, "The chemokine receptor CXCR4 is expressed in pancreatic intraepithelial neoplasia," Gut, vol. 57, no. 11, pp. 1555-1560, 2008.

[88] D. D. Billadeau, S. Chatterjee, P. Bramati et al., "Characterization of the CXCR4 signaling in pancreatic cancer cells," International Journal of Gastrointestinal Cancer, vol. 37, no. 4, pp. 110-119, 2006.

[89] X. Shen, A. Artinyan, D. Jackson, R. M. Thomas, A. M. Lowy, and J. Kim, "Chemokine receptor CXCR4 enhances proliferation in pancreatic cancer cells through AKT and ERK dependent pathways," Pancreas, vol. 39, no. 1, pp. 8187, 2010.

[90] X. Li, Q. Ma, Q. Xu et al., "SDF-1/CXCR4 signaling induces pancreatic cancer cell invasion and epithelial-mesenchymal transition in vitro through non-canonical activation of hedgehog pathway," Cancer Letters, vol. 322, no. 2, pp. 169176, 2012.

[91] Z. Wang, Q. Ma, Q. Liu et al., "Blockade of SDF-1/CXCR4 signalling inhibits pancreatic cancer progression in vitro via inactivation of canonical Wnt pathway," British Journal of Cancer, vol. 99, no. 10, pp. 1695-1703, 2008.

[92] J. Cui, W. Jiang, S. Wang, L. Wang, and K. Xie, "Role of Wnt/beta-catenin signaling in drug resistance of pancreatic cancer," Current Pharmaceutical Design, vol. 18, no. 17, pp. 2464-2471, 2012.

[93] K. P. Olive, M. A. Jacobetz, C. J. Davidson et al., "Inhibition of hedgehog signaling enhances delivery of chemotherapy in a mouse model of pancreatic cancer," Science, vol. 324, no. 5933, pp. 1457-1461, 2009.

[94] S. Singh, S. K. Srivastava, A. Bhardwaj, L. B. Owen, and A. P. Singh, "CXCL12-CXCR4 signalling axis confers gemcitabine resistance to pancreatic cancer cells: a novel target for therapy," British Journal of Cancer, vol. 103, no. 11, pp. 1671-1679, 2010.

[95] U. M. Domanska, R. C. Kruizinga, W. B. Nagengast et al., "A review on CXCR4/CXCL12 axis in oncology: no place to hide," European Journal of Cancer, vol. 49, no. 1, pp. 219230, 2013.

[96] M. Cojoc, C. Peitzsch, F. Trautmann, L. Polishchuk, G. D. Telegeev, and A. Dubrovska, "Emerging targets in cancer management: role of the CXCL12/CXCR4 axis," OncoTargets and Therapy, vol. 6, pp. 1347-1361, 2013.

[97] I. S. Hong, "Stimulatory versus suppressive effects of GMCSF on tumor progression in multiple cancer types," Experimental \& Molecular Medicine, vol. 48, no. 7, article e242, 2016.

[98] L. J. Bayne, G. L. Beatty, N. Jhala et al., "Tumor-derived granulocyte-macrophage colony-stimulating factor regulates myeloid inflammation and $\mathrm{T}$ cell immunity in pancreatic cancer," Cancer Cell, vol. 21, no. 6, pp. 822-835, 2012.

[99] Y. Pylayeva-Gupta, K. E. Lee, C. H. Hajdu, G. Miller, and D. Bar-Sagi, "Oncogenic Kras-induced GM-CSF production promotes the development of pancreatic neoplasia," Cancer Cell, vol. 21, no. 6, pp. 836-847, 2012.

[100] M. Waghray, M. Yalamanchili, M. Dziubinski et al., "GM-CSF mediates mesenchymal-epithelial cross-talk in pancreatic cancer," Cancer Discovery, vol. 6, no. 8, pp. 886899, 2016.

[101] X. X. Wei, S. Chan, S. Kwek et al., "Systemic GM-CSF recruits effector T cells into the tumor microenvironment in localized prostate cancer," Cancer Immunology Research, vol. 4, no. 11, pp. 948-958, 2016.

[102] C. I. Rivas, J. C. Vera, F. Delgado-López et al., "Expression of granulocyte-macrophage colony-stimulating factor receptors in human prostate cancer," Blood, vol. 91, no. 3, pp. 10371043, 1998.

[103] A. Elnemr, T. Ohta, A. Yachie et al., "Human pancreatic cancer cells express non-functional Fas receptors and counterattack lymphocytes by expressing Fas ligand; a potential mechanism for immune escape," International Journal of Oncology, vol. 18, no. 1, pp. 33-39, 2001.

[104] M. Kornmann, T. Ishiwata, J. Kleeff, H. G. Beger, and M. Korc, "Fas and Fas-ligand expression in human pancreatic cancer," Annals of Surgery, vol. 231, no. 3, pp. 368-379, 2000.

[105] T. Ohta, A. Elnemr, H. Kitagawa et al., "Fas ligand expression in human pancreatic cancer," Oncology Reports, vol. 12, no. 4, pp. 749-754, 2004.

[106] Q. Y. Liu, M. A. Rubin, C. Omene, S. Lederman, and C. A. Stein, "Fas ligand is constitutively secreted by prostate cancer cells in vitro," Clinical Cancer Research, vol. 4, no. 7, pp. 1803-1811, 1998.

[107] A. J. Abusamra, Z. Zhong, X. Zheng et al., "Tumor exosomes expressing Fas ligand mediate $\mathrm{CD} 8^{+} \mathrm{T}$-cell apoptosis," Blood Cells, Molecules \& Diseases, vol. 35, no. 2, pp. 169-173, 2005.

[108] M. L. Hyer, C. Voelkel-Johnson, S. Rubinchik, J. Y. Dong, and J. S. Norris, "Intracellular Fas ligand expression causes Fas-mediated apoptosis in human prostate cancer cells resistant to monoclonal antibody-induced apoptosis," Molecular Therapy, vol. 2, no. 4, pp. 348-358, 2000. 


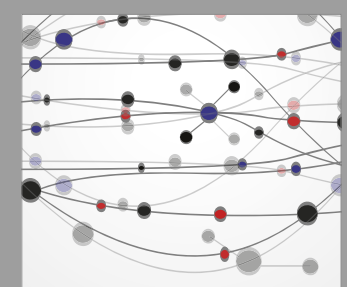

The Scientific World Journal
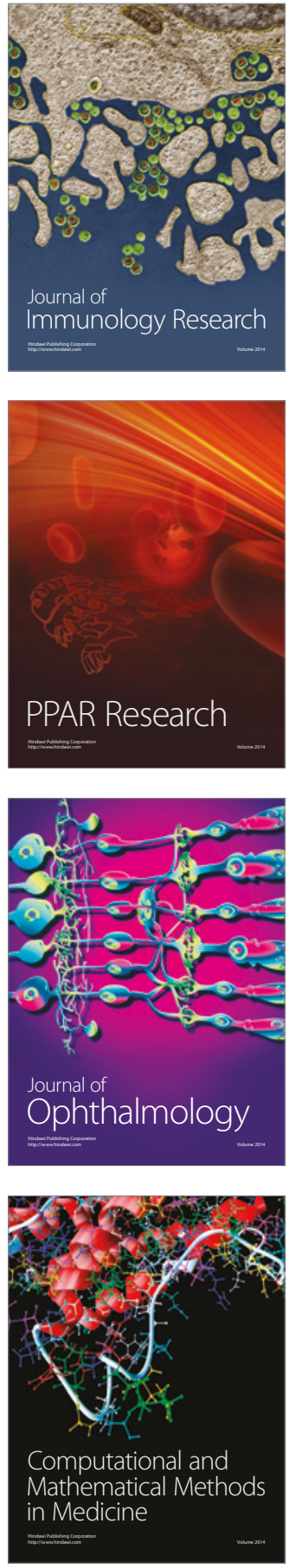

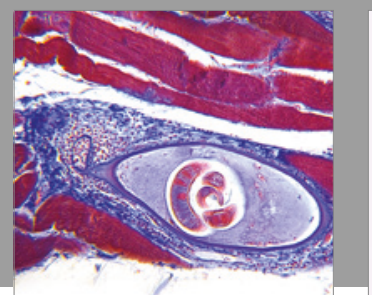

Gastroenterology Research and Practice
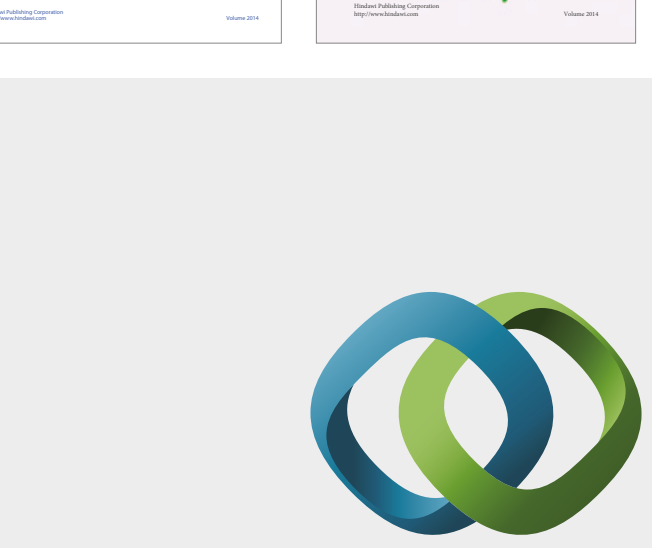

\section{Hindawi}

Submit your manuscripts at

https://www.hindawi.com
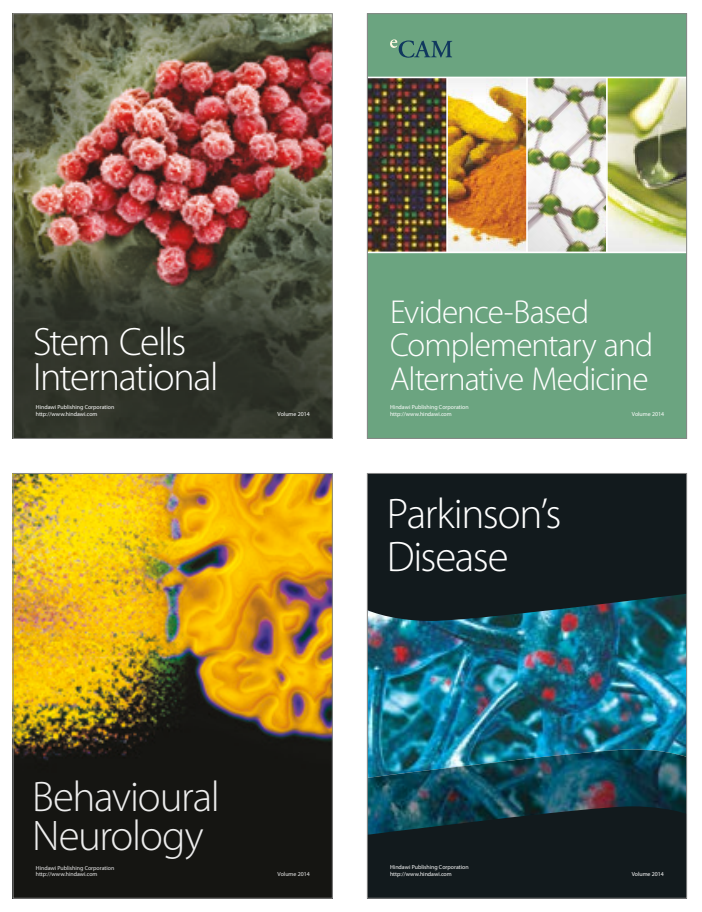
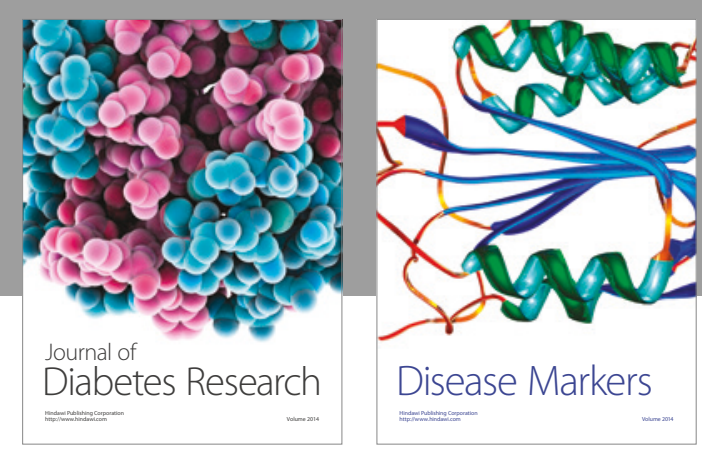

Disease Markers
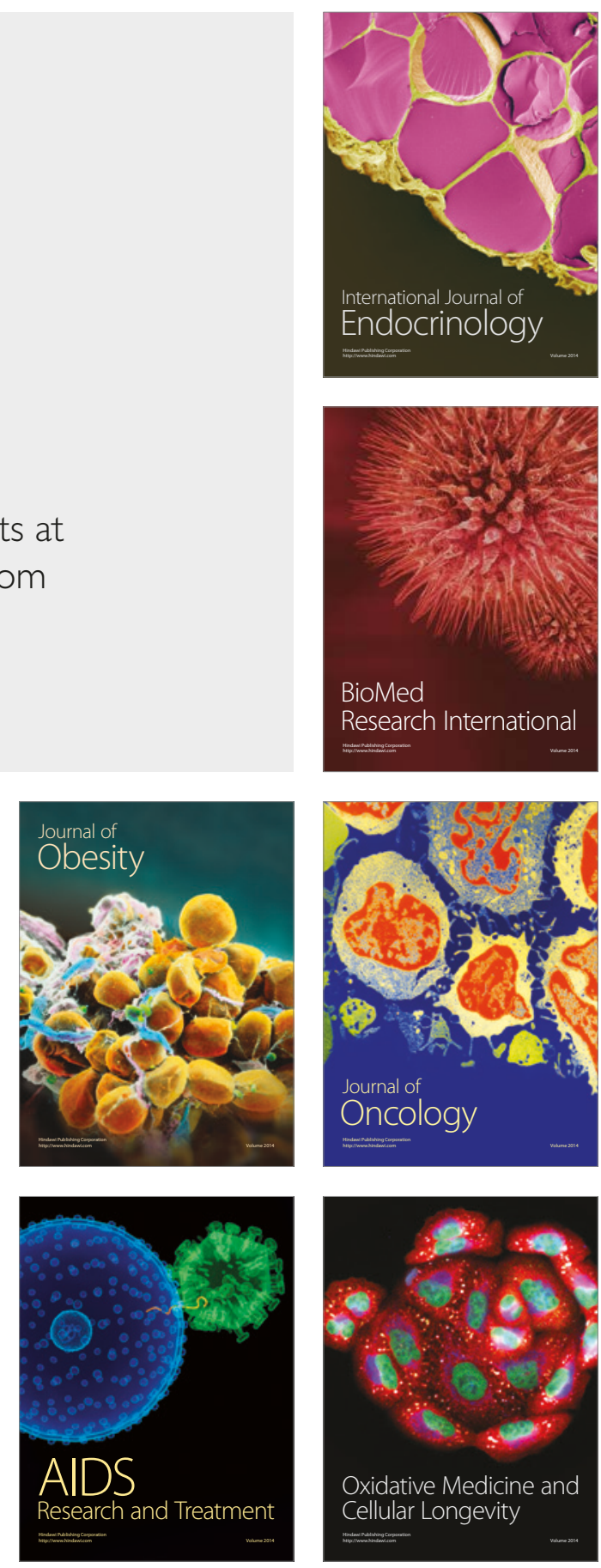